\title{
Atomic Processes of the Solid-Liquid Phase Transition in Metals with Close Packed Structrues
}

\author{
By Sakae Takeuchi* and Hideo Hayasaka**
}

\begin{abstract}
Atomic processes associated with the solid-liquid phase transition in close packed metals are discussed. There exists a definite temperature $T^{*}$ at which the resonance in vibration takes place between atoms adjacent to vacancies and atoms apart from them. A number of Frenkal defects originates in a crystalline domain around a vacancy through the chain-reaction like spreading of the resonance at $T^{*}$, and the domain results in the random arrangement. It becomes more probable to generate vacancies in the surface of crystal at $T^{*}$. The surface layer and the crystalline domain near the surface change into the disorderly arrangement of atoms due to some of surface vacancies, and others diffuse into the disordered domains and the remaining crystalline domains. Vacancies can travel easily in the disordered domain, and this means that there exist atoms in the translational motion, their concentration being identical with vacancies. Liquid is the state in which the vibrational motion coexists with the translational one. The temperature $T^{*}$ is the melting point of metals which is given by $(9 / 8)\left(k T_{\mathrm{m}} / L_{0}\right)=0.0344$. The latent heat of fusion and the entropy change on melting are evaluated in good agreement with the observation according to the above atomic processes.
\end{abstract}

(Received March 27, 1986)

Keywords: anharmonics vibration, resonance in vibration, avalanche like growth of Frenkel defects, vacancy in disorderly arrangement of atoms, coesisting of vibrational motion and translational one, latent heat of fusion and entropy change

\section{Introduction}

Melting is likely to be the only phenomenon in solid state physics in which the atomic process has not been completely understood up to now.

Phenomenological theories including a model such as internal pre-melting disagree with the experimental data. Sharp X-ray diffraction line profiles show that high purity solid argon is a well-behaved periodic crystal at temperature within $0.1^{\circ}$ of the triple point ${ }^{(1)}$. A similar result has been observed also in the case of high purity silver. The difficulties in the classical theory of phase transition have stimulated an alternative approach to the melting problem associating with the formation of defects such as vacancies, interstitials or dislocations ${ }^{(2)-(4)}$.

* Emeritus Professor of Tohoku University, The Research Institute for Iron, Steel and Other Metals, Tohoku University, Sendai 980, Japan.

** Radiation Engineering Laboratory, Faculty of Engineering, Tohoku University, Sendai 980, Japan.
The bulk coefficient of thermal expansion in liquid $\alpha_{\mathrm{L}}$ is fairly larger than that in solid $\alpha_{\mathrm{s}},-$ for $\mathrm{Cu} \alpha_{\mathrm{s}}=0.051$ and $\alpha_{\mathrm{L}}=0.100$ in the unit of $\left(\mathrm{cm}^{3} / \mathrm{mol}\right) \cdot \mathrm{deg}^{-1} \times 10^{3}-$, and the specific heat $C_{\mathrm{V}}$ is nearly $3 \mathrm{Nk}$ at the melting temperature, however it decreases with increasing temperature. The large volume change $(\Delta V / V)_{\mathrm{m}}$ on melting is observed in a range of $0.06 \sim 0.04$ in f.c.c. metals and of 0.1 in solid $\mathrm{Ar}$ and $\mathrm{Kr}$. These thermal properties of liquid can be understood by reflecting the contribution of vacancies or voids with the atomic size.

The vacancy concentration in crystals increases rapidly in a higher temperature range near the melting point $T_{\mathrm{m}}$, and the equilibrium vacancy concentration $(\Delta N / N)_{\mathrm{m}}$ in solid at $T_{\mathrm{m}}$ is the order of $10^{-4}$ in metals with f.c.c. structure and of $10^{-3}$ in solid Ar or $\mathrm{Kr}$. It has been shown experimentally by Simons ${ }^{(5)}$ that for f.c.c. metals $\mathrm{Ag}, \mathrm{Cu}, \mathrm{Au}$ and $\mathrm{Al}$, the fractional volume change on melting $(\Delta V / V)_{\mathrm{m}}$ is approximately proportional to the equilibrium vacancy cncentration $(\Delta N / N)_{\mathrm{m}}$ in solid at $T_{\mathrm{m}}$. The formula proposed by Simons can be rewritten as follows, 


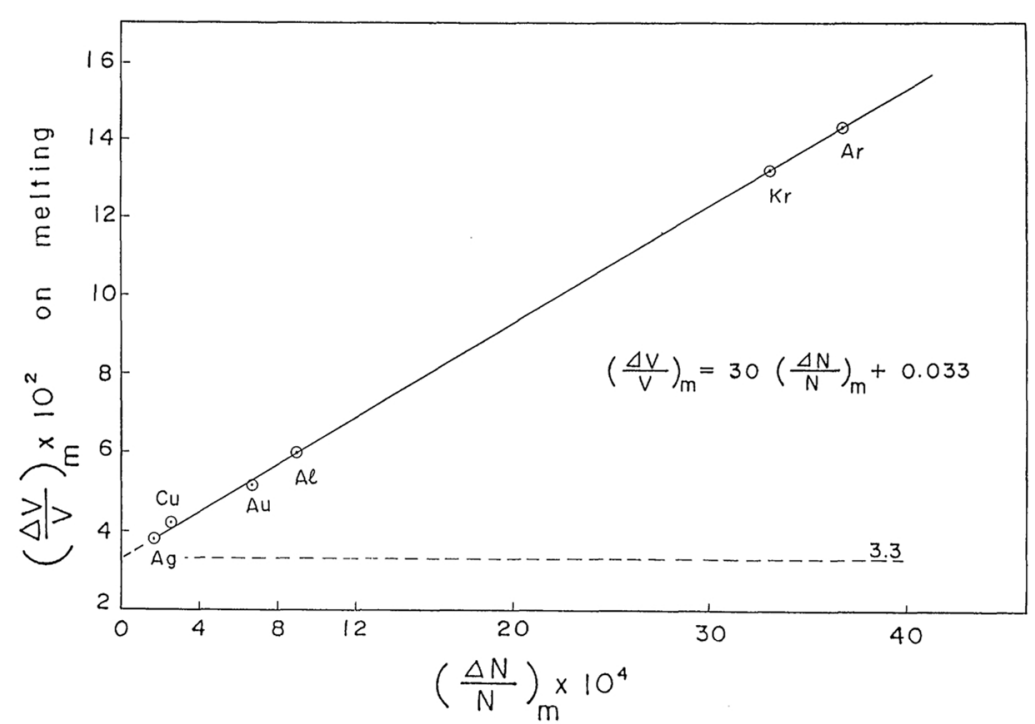

Fig. 1 Relation between volume change and vacancy concentration at melting point.

$$
\left(\frac{\Delta V}{V}\right)_{\mathrm{m}}=30\left(\frac{\Delta N}{N}\right)_{\mathrm{m}}+0.033
$$

This relation can be extended up to solid $\mathrm{Ar}$ and $\mathrm{Kr}$ as shown in Fig. 1. Equation (1) suggests that the first term on the right-hand side corresponds to the vacancy concentration for the transition from the ordered state in crystal to the disorderly arrangement of atoms and the second term for bringing about fluidity through the traveling of atoms in the disordered state. If we put from eq. (1)

$$
l=\frac{1}{30\left(\frac{\Delta N}{N}\right)_{\mathrm{m}}},
$$

it is supposed that $30(\Delta N)_{\mathrm{m}}$ vacancies introduced into a crystal upon melting play a role in causing the transition by which a crystalline domain of $l$ atoms per a vacancy on the average changes into the disordered arrangement. Such a vacancy mechanism of melting is understood from the behaviour of atoms under the anharmonic vibration in the crystal lattice including vacancies.

At first, the anharmonic vibrations of atom $y$ adjoining a vacancy and of atom $x$ apart from it in the crystal lattice are discussed in the section II and III. Shift of atom $y$ toward the vacancy originating from the anharmonic motion increases with increasing temperature, and hence the temperature dependence of the fre- quency $\omega_{y}$ of atom $y$ becomes negative on the contrary to that of the frequency $\omega_{\mathrm{x}}$ of atom $x$. Thus it is understood that there exists a definite temperature $T^{*}$ at which the condition for the resonance in vibration $\omega_{\mathrm{y}}\left(T^{*}\right)=\omega_{\mathrm{x}}\left(T^{*}\right)$ is satisfied. In the section IV, the atomic processes of melting are developed by considering phenomena brought about by the resonance in vibration. As a result of the resonance in vibration at $T^{*}$, atom $y$ is displaced to an interstitial position of the lattice leaving a vacant site behind and then any other atom neighbouring the vacancy formed by the displacement of atom $y$ shifts to the other interstitial position. Such a process will successively be carried out. Through these chain-reactions many Frenkel pairs are formed over a crystalline domain around a vacancy in avalanche-like wise. When the concentration of Frenkel defect increases over about $40 \%$ in a domain with f.c.c. structure, its crystalline state changes into the random arrangement like an amorphous state $^{(6)}$. It becomes more probable to generate vacancies in the surface layer at $T^{*}$ where atoms are supposed to be in a similar circumstance to atoms adjoining vacancies in the lattice. These surface vacancies diffuse into the inner part of lattice. In the disordered domain atoms can travel easily through the jumping into neighbouring vacancies or voids flowed from the surface. This jumping process means 
that the vibrational motion of atoms adjoing vacancies changes into the translational one. Liquid is the state in which the vibrational motion coexists with the translational one. Consequently the temperature $T^{*}$ at which the resonance takes place in the atomic vibration can be suposed to be the melting point of metal $T_{\mathrm{m}}$. A simple relation between the cohesive energy of metal $L_{0}$ and its melting temperature $T_{\mathrm{m}}$,

$$
\frac{9}{8} \frac{k T_{\mathrm{m}}}{L_{0}}=0.0344,
$$

is obtained from the condition for the resonance.

In the section $\mathrm{V}$, the latent heat of fusion $\Delta E$ and the entropy change $\Delta S$ are calculated independent of each other using the above relation in good agreement with the observation for many metals with f.c.c. and h.c.p. structure.

\section{Anharmonic Behaviours of Atomic Vibration in Crystal Lattices}

Atomic vibrations in a crystal are considered to be anharmonic in a temperature range near the melting point. Anharmonic behaviours of vibration of atoms occupying lattice sites along the [110] direction in the f.c.c. lattice are discussed.

The potential energy of atom $n$ due to interactions between nearest neighbours $n-1$ and $n+1$ is written as

$$
\begin{aligned}
V\left(x_{n}\right)= & -2 V_{0}+\frac{\kappa}{2}\left[\left(x_{n-1}-x_{n}\right)^{2}+\left(x_{n}-x_{n+1}\right)^{2}\right] \\
& +\frac{\alpha}{3}\left[\left(x_{n-1}-x_{n}\right)^{3}+\left(x_{n}-x_{n+1}\right)^{3}\right]+\cdots,
\end{aligned}
$$

where $x_{n-1}, x_{n}$ and $x_{n+1}$ are the deflections of atoms $n-1, n$ and $n+1$ from their equilibrium positions. The equation of motion of atom $n$ is given, then, as follows,

$$
\begin{aligned}
\ddot{x}_{n} & +\Omega_{0}^{2}\left(2 x_{n}-x_{n-1}-x_{n+1}\right) \\
& =\theta_{0}^{2}\left(2 x_{n}-x_{n-1}-x_{n+1}\right)\left(x_{n+1}-x_{n-1}\right),
\end{aligned}
$$

where $\Omega_{0}^{2}=\kappa / m, \theta_{0}^{2}=\alpha / m$, and $m$ is the mass of atom. To know the anharmonic behaviour of the motion of atom $n$ from eq. (3), we put

$$
\begin{array}{ll}
x_{n}=x_{n}^{(0)}+x_{n}^{(1)}+x_{n}^{(2)}+\cdots, & x_{n}^{(0)}>x_{n}^{(1)}>x_{n}^{(2)}, \\
x_{n-1}=x_{n-1}^{(0)}+x_{n-1}^{(1)}+x_{n-1}^{(2)}+\cdots, & x_{n-1}^{(0)}>x_{n-1}^{(1)}>x_{n-1}^{(2)}, \\
x_{n+1}=x_{n+1}^{(0)}+x_{n+1}^{(1)}+x_{n+1}^{(2)}+\cdots, & x_{n+1}^{(0)}>x_{n+1}^{(1)}>x_{n+1}^{(2)} .
\end{array}
$$

Equation (3) is then rewritten approximately as follows,

$$
\begin{aligned}
& \ddot{x}_{n}^{(0)}+\ddot{x}_{n}^{(1)}+\ddot{x}_{n}^{(2)}+\Omega_{0}^{2}\left(2 x_{n}^{(0)}-x_{n-1}^{(0)}-x_{n+1}^{(0)}\right)+\Omega_{0}^{2}\left(2 x_{n}^{(1)}-x_{n-1}^{(1)}-x_{n+1}^{(1)}\right)+\Omega_{0}^{2}\left(2 x_{n}^{(2)}-x_{n-1}^{(2)}-x_{n+1}^{(2)}\right) \\
= & \theta_{0}^{2}\left[\left(2 x_{n}^{(0)}-x_{n-1}^{(0)}-x_{n+1}^{(0)}\right)\left\{\left(x_{n+1}^{(0)}-x_{n-1}^{(0)}\right)+\left(x_{n+1}^{(1)}-x_{n-1}^{(1)}\right)+\left(x_{n+1}^{(2)}-x_{n-1}^{(2)}\right)\right\}\right. \\
& +\left(2 x_{n}^{(1)}-x_{n-1}^{(1)}-x_{n+1}^{(1)}\right)\left\{\left(x_{n+1}^{(0)}-x_{n-1}^{(0)}\right)+\left(x_{n+1}^{(1)}-x_{n-1}^{(1)}\right)\right\} \\
& \left.+\left(2 x_{n}^{(2)}-x_{n-1}^{(2)}-x_{n+1}^{(2)}\right)\left(x_{n+1}^{(0)}-x_{n-1}^{(0)}\right)\right] .
\end{aligned}
$$

We may try to solve this eq. (4) by a running wave of type

$$
\begin{array}{lll}
x_{n}^{(0)}=A \sin \omega t, & x_{n-1}^{(0)}=A \sin (\omega t-q a), & x_{n+1}^{(0)}=A \sin (\omega t+q a) \\
x_{n}^{(1)}=B \sin 2 \omega t, & x_{n-1}^{(1)}=B \sin (2 \omega t-2 q a), & x_{n+1}^{(1)}=B \sin (2 \omega t+2 q a) \\
x_{n}^{(2)}=C \sin 3 \omega t, & x_{n-1}^{(2)}=C \sin (3 \omega t-3 q a), & x_{n+1}^{(2)}=C \sin (3 \omega t+3 q a),
\end{array}
$$

where $q=(2 \pi / \lambda)$ is the wave vector and $a$ is the equilibrium distance between neibouring atoms. Substituting these solutions of (5) for the differential equation (4), one obtains

$$
\begin{aligned}
& \left\{-\omega^{2}+4 \Omega_{0}^{2} \delta_{s}^{2}-16 B \theta_{0}^{2} \delta_{s}^{3} \delta_{c}\right\} A \sin \omega t \\
& \quad+\left\{-4 \omega^{2}+16 \Omega_{0}^{2} \delta_{s}^{2} \delta_{c}^{2}-8 \frac{A^{2}}{B} \theta_{0}^{2} \delta_{s}^{3} \delta_{c}+16 \frac{A C}{B} \theta_{0}^{2}\left(1-4 \delta_{c}^{2}\right) \delta_{s}^{3} \delta_{c}\right\} B \sin 2 \omega t \\
& \quad+\left\{-9 \omega^{2}+4 \Omega_{0}^{2}\left(1-4 \delta_{c}^{2}\right)^{2} \delta_{s}^{2}+16 \frac{A B}{C} \theta_{0}^{2}\left(1-4 \delta_{c}^{2}\right) \delta_{s}^{3} \delta_{c}\right\} C \sin 3 \omega t=0,
\end{aligned}
$$


where $\delta_{\mathrm{s}}$ and $\delta_{\mathrm{c}}$ are the parameters relating to the phase shift in vibration $q a$ and are given by

$$
\delta_{\mathrm{s}}=\sin \frac{q a}{2} \quad \text { and } \quad \delta_{\mathrm{c}}=\cos \frac{q a}{2} \text {. }
$$

However, we can have the following relations from the above eq. (6) because of no interference between $\sin \omega t, \sin 2 \omega t$ and $\sin 3 \omega t$,

$$
\begin{aligned}
& \frac{\omega^{2}}{\Omega_{0}^{2}}=4 \delta_{s}^{2}-16 B \frac{\theta_{0}^{2}}{\Omega_{0}^{2}} \delta_{s}^{3} \delta_{c}, \\
& 4 \frac{\omega^{2}}{\Omega_{0}^{2}}=16 \delta_{s}^{2} \delta_{c}^{2}-8 \frac{A^{2}}{B} \frac{\theta_{0}^{2}}{\Omega_{0}^{2}} \delta_{s}^{3} \delta_{c}+16 \frac{A C}{B} \frac{\theta_{0}^{2}}{\Omega_{0}^{2}}\left(1-4 \delta_{c}^{2}\right) \delta_{s}^{3} \delta_{c}, \\
& 9 \frac{\omega^{2}}{\Omega_{0}^{2}}=4\left(1-4 \delta_{c}^{2}\right)^{2} \delta_{s}^{2}+16 \frac{A B}{C} \frac{\theta_{0}^{2}}{\Omega_{0}^{2}}\left(1-4 \delta_{c}^{2}\right) \delta_{s}^{3} \delta_{c} .
\end{aligned}
$$

On the understanding of the above relations (8) eq. (4) is equivalent to the equation

$$
\left(\ddot{x}_{n}^{(0)}+\omega^{2} x_{n}^{(0)}\right)+\left(\ddot{x}_{n}^{(1)}+4 \omega^{2} x_{n}^{(1)}\right)+\left(\ddot{x}_{n}^{(2)}+9 \omega^{2} x_{n}^{(2)}\right)=0 .
$$

The following relations are obtained by eliminating $\omega^{2} / \Omega_{0}^{2}$ from (8)

$$
\begin{aligned}
2 \frac{\delta_{s}}{\delta_{c}} & =8 B \frac{\theta_{0}^{2}}{\Omega_{0}^{2}}-\frac{A^{2}}{B} \frac{\theta_{0}^{2}}{\Omega_{0}^{2}}+2 \frac{A C}{B} \frac{\theta_{0}^{2}}{\Omega_{0}^{2}}\left(1-4 \delta_{c}^{2}\right) \\
2 \frac{\delta_{s}}{\delta_{c}}\left(1+2 \delta_{c}^{2}\right) & =9 B \frac{\theta_{0}^{2}}{\Omega_{0}^{2}}+\frac{A B}{C} \frac{\theta_{0}^{2}}{\Omega_{0}^{2}}\left(1-4 \delta_{c}^{2}\right) .
\end{aligned}
$$

$B$ and $C$ can be calculated in terms of $A$ and $\delta$ from (10) and (11). After some manipulation $B$ is given approximately in the following form

$$
B=\frac{1}{8} \frac{\Omega_{0}^{2}}{\theta_{0}^{2}} \frac{\delta_{s}}{\delta_{c}}-\frac{1}{32 \sqrt{2}} \frac{1}{A} \frac{\Omega_{0}^{4}}{\theta_{0}^{4}} \frac{\delta_{s}^{2}}{\delta_{c}^{2}}-\frac{1}{2 \sqrt{2}}\left\{1-\frac{1}{16} \frac{\left(1-4 \delta_{c}^{2}\right)^{2}}{1+2 \delta_{c}^{2}}\right\} A-\frac{1}{16} A^{2} \frac{\theta_{0}^{2}}{\Omega_{0}^{2}} \frac{\left(1-4 \delta_{c}^{2}\right)^{2}}{1+2 \delta_{c}^{2}} \frac{\delta_{c}}{\delta_{s}} .
$$

$C$ is obtained from (10)

$$
C=\frac{1}{2 \frac{\theta_{0}^{2}}{\Omega_{0}^{2}}\left(1-4 \delta_{c}^{2}\right)}\left(A \frac{\theta_{0}^{2}}{\Omega_{0}^{2}}-8 \frac{B^{2}}{A} \frac{\theta_{0}^{2}}{\Omega_{0}^{2}}+2 \frac{B}{A} \frac{\delta_{s}}{\delta_{c}}\right) .
$$

Substituting (12) for $B$ in (8-a) $\omega^{2} / \Omega_{0}^{2}$ is also given in terms of $A$ and $\delta$ as follows.

$$
\frac{\omega^{2}}{\Omega_{0}^{2}}=4 \delta_{s}^{2}-2 \delta_{s}^{4}+\frac{1}{2 \sqrt{2}} \frac{\delta_{s}^{5}}{\delta_{c}} \frac{1}{A \frac{\theta_{0}^{2}}{\Omega_{0}^{2}}}+\frac{8}{\sqrt{2}}\left\{1-\frac{1}{16} \frac{\left(1-4 \delta_{c}^{2}\right)^{2}}{1+2 \delta_{c}^{2}}\right\} \delta_{s}^{3} \delta_{c} A \frac{\theta_{c}^{2}}{\Omega_{0}^{2}}+\frac{\left(1-4 \delta_{c}^{2}\right)^{2}}{1+2 \delta_{c}^{2}} \delta_{s}^{2} \delta_{c}^{2} A^{2} \frac{\theta_{0}^{4}}{\Omega_{0}^{4}} .
$$

It is necessary to know the temperature variation of $\omega^{2} / \Omega_{0}^{2}$ in the high temperature range near the melting point for understanding the anharmonic behaviour in the atomic vibration. The vibrational energy $E$ of atom $n$ can be given from (9) by

$$
E=m \omega^{2}\left(\frac{A^{2}}{2}+2 B^{2}+\frac{9}{2} C^{2}\right),
$$

and then according to the law of equipartition we have

$$
\frac{\omega^{2}}{\Omega_{0}^{2}}\left(\frac{A^{2}}{2}+2 B^{2}+\frac{9}{2} C^{2}\right)=\frac{k T}{m \Omega_{0}^{2}} .
$$

$\omega^{2} / \Omega_{0}^{2}$ and $B^{2}$ in (15) are given in terms of $A$ from (14) and (12), respectively. The term of $C^{2}$ can be neglected practically because of $A^{2} \gg B^{2} \gg C^{2}$. The temperature dependence of $A \theta_{0}^{2} / \Omega_{0}^{2}$ 
calculated from (15), (14) and (12) is given approximately by

where

$$
\begin{aligned}
A \frac{\theta_{0}^{2}}{\Omega_{0}^{2}}= & \frac{1}{6 \sqrt{2}} \frac{\delta_{s}}{\delta_{c}} \frac{\left(1-\frac{11}{8} \delta_{s}^{2}\right)}{\alpha-\frac{5}{6} \beta \delta_{s}^{2}}+\frac{1}{\sqrt{3}} \frac{1}{\delta_{s}} \sqrt{\frac{\xi}{\alpha-\frac{5}{6} \beta \delta_{s}^{2}}} \\
& -\frac{1}{8 \sqrt{3}} \frac{\delta_{s}^{3}}{\delta_{c}^{2}}\left\{\left(1-\frac{3}{4} \delta_{s}^{2}\right)-\frac{1}{6} \frac{\left(1-\frac{11}{8} \delta_{s}^{2}\right)}{\alpha-\frac{5}{6} \beta \delta_{s}^{2}}\right\} \frac{1}{\sqrt{\left(\alpha-\frac{5}{6} \beta \delta_{s}^{2}\right) \cdot \xi}},
\end{aligned}
$$

$$
\left.\begin{array}{l}
\xi=\frac{k T}{m \Omega_{0}^{2}} \frac{\theta_{0}^{4}}{\Omega_{0}^{4}}, \\
\alpha=1-\frac{1}{12} \frac{\left(1-4 \delta_{c}^{2}\right)^{2}}{1+2 \delta_{c}^{2}}\left(1-\frac{9}{16} \frac{1}{1+2 \delta_{c}^{2}}\right), \\
\beta=1-\frac{7}{80} \frac{\left(1-4 \delta_{c}^{2}\right)^{2}}{1+2 \delta_{c}^{2}}\left(1-\frac{27}{56} \frac{1}{1+2 \delta_{c}^{2}}\right) .
\end{array}\right\}
$$

The quantity $\delta_{s}$ or $\delta_{\mathrm{c}}$ relating to the phase shift in vibration is included as a parameter in eqs. (12), (14) and (16). However, $\delta_{\mathrm{s}}$ or $\delta_{\mathrm{c}}$ depends upon temperature. It is desired to search for a relation between $\delta_{s}$ and $\xi$. One obtains the following relation among $\delta_{s}, A \theta_{0}^{2} / \Omega_{0}^{2}$ and $B \theta_{0}^{2} / \Omega_{0}^{2}$ by eliminating $C$ from (10) and (11),

$$
2 \frac{\delta_{s}}{\delta_{c}}=8 B \frac{\theta_{0}^{2}}{\Omega_{0}^{2}}-\frac{A^{2} \frac{\theta_{0}^{4}}{\Omega_{0}^{4}}}{B \frac{\theta_{0}^{2}}{\Omega_{0}^{2}}}+\frac{\left(1-4 \delta_{c}^{2}\right)^{2} A^{2} \frac{\theta_{0}^{4}}{\Omega_{0}^{4}}}{\frac{\delta_{s}}{\delta_{c}}\left(1+2 \delta_{c}^{2}\right)-\frac{9}{2} B \frac{\theta_{0}^{2}}{\Omega_{0}^{2}}} .
$$

$A \theta_{0}^{2} / \Omega_{0}^{2}$ in (16) and $B \theta_{0}^{2} / \Omega_{0}^{2}$ in (12) are functions of $\delta_{\mathrm{s}}$ and $\xi$, but they have been obtained approximetely neglecting amall quantities. We define a quantity $\Delta$ from eq. (18) as follows,

$$
\Delta=2 \frac{\delta_{s}}{\delta_{c}}-\left[8 B \frac{\theta_{0}^{2}}{\Omega_{0}^{2}}-\frac{A^{2} \frac{\theta_{0}^{4}}{\Omega_{0}^{4}}}{B \frac{\theta_{0}^{2}}{\Omega_{0}^{2}}}+\frac{\left(1-4 \delta_{c}^{2}\right)^{2} A^{2} \frac{\theta_{0}^{4}}{\Omega_{0}^{4}}}{\frac{\delta_{s}}{\delta_{c}}\left(1+2 \delta_{c}^{2}\right)-\frac{9}{2} B \frac{\theta_{0}^{2}}{\Omega_{0}^{2}}}\right] .
$$

$A \theta_{0}^{2} / \Omega_{0}^{2}$ and $B \theta_{0}^{2} / \Omega_{0}^{2}$ are calculated numerically as functions of $\xi$ from (16) and (12) for a given value of $\delta_{s}$, and $\Delta$ is plotted against $\xi$ for each of given values of $\delta_{s}$, as shown in Fig. 2. One can search for $\xi$ at which $\Delta$ takes a minimum value on each curve of $\Delta$ for a given value of $\delta_{\mathrm{s}}$. Pairs of $\delta_{\mathrm{s}}$ and $\xi$ obtained in the range of $\xi>0.03$ by the above way are given in the following Tale 1 . The values of $\delta_{\mathrm{s}}$ are estimated within error of about 0.02 in the range from $1 / 4.7$ to $1 / 4.8$ of $\delta_{s}^{2}$, as seen from Fig. 2 .

Temperature dependence of $\omega^{2} / \Omega_{0}^{2}$ is calculated from (14) and (16) by making use of $\delta_{\mathrm{s}}$ and $\xi$ in Table 1 . The plot of $\omega^{2} / \Omega_{0}^{2}$ against $\xi$ is shown in Fig. 3.

\section{Vibrational Behaviours of an Atom Adjacent to a Vacant Site in a Lattice}

Anharmonic vibrations of an atom adjoining a vacant site in the direction [110] of the f.c.c. lattice are discussed. If the deflections from the equilibrium position of atom 1 ajoining the vacant site and of atom 2 apart from it are denoted by $y$ and $x$, respectively, in the [110] direction of the lattice, as shown in Fig. 4, the potential energy of atom 1 is given by 


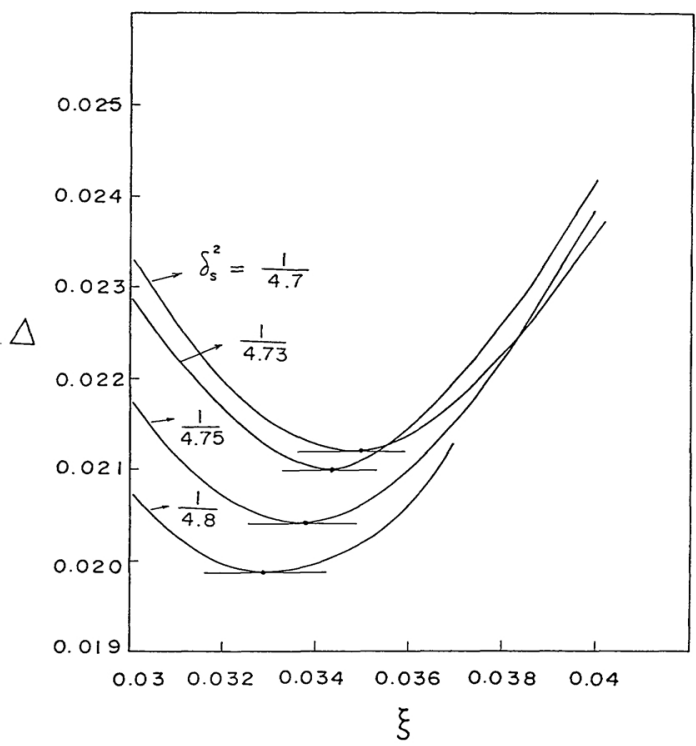

Fig. 2 Temperature dependence of minimum position in $\Delta$ with various values of $\delta_{s}^{2}$.

Table 1 Temperature dependence of $\delta_{s}^{2}$ and $\left(\omega^{2} / \Omega_{0}^{2}\right)$. $\left(\xi=\left(k T / m \Omega_{0}^{2}\right)\left(\theta_{0}^{4} / \Omega_{0}^{4}\right)\right)$

\begin{tabular}{ccc}
\hline \hline \multicolumn{1}{c}{$\delta_{\mathrm{s}}^{2}$} & $\xi$ & $\omega^{2} / \Omega_{0}^{2}$ \\
\hline $1 / 4.7$ & 0.035 & 0.944 \\
$1 / 4.73$ & 0.0344 & 0.937 \\
$1 / 4.75$ & 0.0339 & 0.933 \\
$1 / 4.8$ & 0.0324 & 0.921 \\
\hline \hline
\end{tabular}

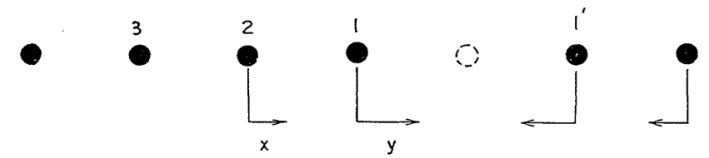

Fig. 4 Coordinates of atoms in the direction [111] of f.c.c. lattice including a vacant site.

$V(y)=-V_{0}+\frac{\kappa}{2}(x-y)^{2}+\frac{\alpha}{3}(x-y)^{3}+\cdots$.

The equation of motion of atom 1 is then

$$
\ddot{y}+\Omega_{0}^{2} y=\theta_{0}^{2} y^{2}-2 \theta_{0}^{2} x y+\Omega_{0}^{2} x+\theta_{0}^{2} x^{2},
$$

where

$$
\Omega_{0}^{2}=\frac{\kappa}{m} \quad \text { and } \quad \theta_{0}^{2}=\frac{\alpha}{m} .
$$

At first, we discuss the behaviours of atom 1 in the case when atom 2 rests on its lattice site. In this case the equation of motion is

$$
\ddot{y}+\Omega_{0}^{2} y=\theta_{0}^{2} y^{2} .
$$

We put

$$
y=\eta+y_{0}+y_{1}+y_{2},
$$

where $\eta$ is an unknown constant independent of time $t$ and $y_{0}>y_{1}>y_{2}$. The equation of motion (23) is rewritten neglecting small quantities $y_{1} y_{2}$ and $y_{2}^{2}$ as follows.

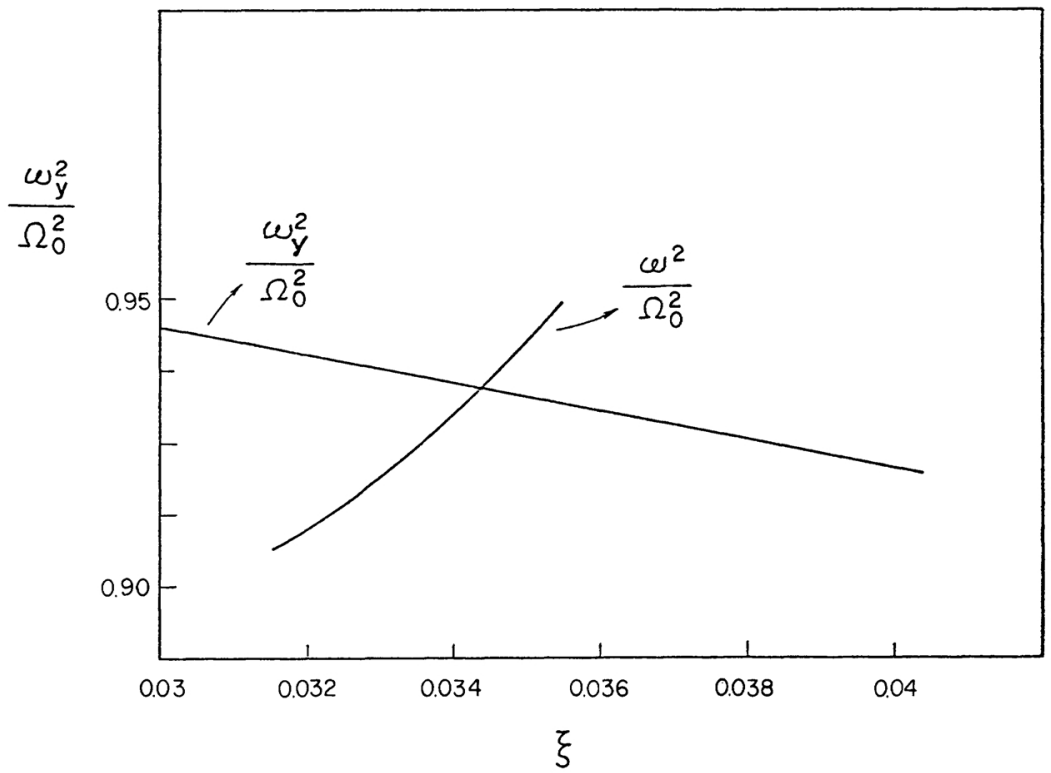

Fig. 3 Temperature dependence of $\left(\omega_{y}^{2} / \Omega_{0}^{2}\right)$ and $\left(\omega^{2} / \Omega_{0}^{2}\right)$. 


$$
\ddot{y}_{0}+\ddot{y}_{1}+\ddot{y}_{2}+\Omega_{0}^{2}\left(y_{0}+y_{1}+y_{2}\right)+\Omega_{0}^{2} \eta=\theta_{0}^{2}\left\{\eta^{2}+y_{0}^{2}+y_{1}^{2}+2 \eta\left(y_{0}+y_{1}+y_{2}\right)+2 y_{0} y_{1}+2 y_{0} y_{2}\right\} \text {. }
$$

We try to solve eq. (25) by putting

$$
y_{0}=A_{y} \cos \omega_{y} t, \quad y_{1}=B_{y} \cos 2 \omega_{y} t, \quad y_{2}=C_{y} \cos 3 \omega_{y} t .
$$

Substituting (26) for the above eq. (25), we have

$$
\begin{aligned}
& \Omega_{0}^{2} \eta-\theta_{0}^{2} \eta^{2}-\frac{1}{2} \theta_{0}^{2}\left(A_{y}^{2}+B_{y}^{2}\right)+\left(\Omega_{0}^{2}-\omega_{y}^{2}-2 \theta_{0}^{2} \eta-\theta_{0}^{2} B_{y}\right) A_{y} \cos \omega_{y} t \\
& +\left(\Omega_{0}^{2}-4 \omega_{y}^{2}-2 \theta_{0}^{2} \eta-\frac{1}{2} \theta_{0}^{2} \frac{A_{y}^{2}}{B_{y}}-\theta_{0}^{2} \frac{A_{y} C_{y}}{B_{y}}\right) B_{y} \cos 2 \omega_{y} t \\
& +\left(\Omega_{0}^{2}-9 \omega_{y}^{2}-2 \theta_{0}^{2} \eta-\theta_{0}^{2} \frac{A_{y} B_{y}}{C_{y}}\right) C_{y} \cos 3 \omega_{y} t=0 .
\end{aligned}
$$

Then, it follows that

$$
\begin{aligned}
& \frac{\theta_{0}^{2}}{\Omega_{0}^{2}} \eta^{2}-\eta+\frac{1}{2} \frac{\theta_{0}^{2}}{\Omega_{0}^{2}}\left(A_{y}^{2}+B_{y}^{2}\right)=0, \\
& \frac{\omega_{y}^{2}}{\Omega_{0}^{2}}=1-2 \frac{\theta_{0}^{2}}{\Omega_{0}^{2}} \eta-\frac{\theta_{0}^{2}}{\Omega_{0}^{2}} B_{y}, \\
& 4 \frac{\omega_{y}^{2}}{\Omega_{0}^{2}}=1-2 \frac{\theta_{0}^{2}}{\Omega_{0}^{2}} \eta-\frac{1}{2} \frac{\theta_{0}^{2}}{\Omega_{0}^{2}} \frac{A_{y}^{2}}{B_{y}}-\frac{\theta_{0}^{2}}{\Omega_{0}^{2}} \frac{A_{y} C_{y}}{B_{y}}, \\
& 9 \frac{\omega_{y}^{2}}{\Omega_{0}^{2}}=1-2 \frac{\theta_{0}^{2}}{\Omega_{0}^{2}} \eta-\frac{\theta_{0}^{2}}{\Omega_{0}^{2}} \frac{A_{y} B_{y}}{C_{y}} .
\end{aligned}
$$

On the understanding of (27) and (28) the equation of motion (25) is rewritten as

$$
\left(\ddot{y}_{0}+\omega_{y}^{2} y_{0}\right)+\left(\ddot{y}_{1}+4 \omega_{y}^{2} y_{1}\right)+\left(\ddot{y}_{2}+9 \omega_{y}^{2} y_{2}\right)=0
$$

One obtains from (27)

$$
\eta=\frac{1}{2} A_{y}^{2} \frac{\theta_{0}^{2}}{\Omega_{0}^{2}}+\frac{1}{4} A_{y}^{4}\left(\frac{\theta_{0}^{4}}{\Omega_{0}^{4}}\right) \frac{\theta_{0}^{2}}{\Omega_{0}^{2}},
$$

from (28)

$$
\begin{aligned}
& B_{y}=-\frac{1}{6} A_{y}^{2} \frac{\theta_{0}^{2}}{\Omega_{0}^{2}}-\frac{7}{54} A_{y}^{4}\left(\frac{\theta_{0}^{4}}{\Omega_{0}^{4}}\right) \frac{\theta_{0}^{2}}{\Omega_{0}^{2}}, \\
& C_{y}=\frac{1}{48} A_{y}\left(A_{y}^{2} \frac{\theta_{0}^{4}}{\Omega_{0}^{4}}\right),
\end{aligned}
$$

and from (28), (30) and (31)

$$
\frac{\omega_{y}^{2}}{\Omega_{0}^{2}}=1-\frac{5}{6} A_{y}^{2} \frac{\theta_{0}^{4}}{\Omega_{0}^{4}}-\frac{10}{27} A_{y}^{4}\left(\frac{\theta_{0}^{4}}{\Omega_{0}^{4}}\right)^{2} .
$$

The following equation is obtained from (29) and the law of equipartition

$$
\frac{\omega_{y}^{2}}{\Omega_{0}^{2}}\left(\frac{A_{y}^{2}}{2}+2 B_{y}^{2}+\frac{9}{2} C_{y}^{2}\right)=\frac{k T}{m \Omega_{0}^{2}} .
$$

Substituting (31) and (32) for (33) $A_{y}^{2}$ is solved in terms of temperature $T$.

$$
A_{y}^{2}=2 \frac{k T}{m \Omega_{0}^{2}}+\frac{26}{9}\left(\frac{k T}{m \Omega_{0}^{2}}\right)^{2} \frac{\theta_{0}^{4}}{\Omega_{0}^{4}} .
$$

Thus we have the following formula depending upon temperature for $\omega_{y}^{2} / \Omega_{0}^{2}$ of an atom adjoining a vacancy in a lattice by substituting (34) for (32).

$$
\frac{\omega_{y}^{2}}{\Omega_{0}^{2}}=1-\frac{5}{3} \xi-\frac{35}{9} \xi^{2}-\frac{1040}{243} \xi^{3},
$$

where

$$
\xi=\frac{k T}{m \Omega_{0}^{2}} \frac{\theta_{0}^{4}}{\Omega_{0}^{4}} .
$$

The plott of $\omega_{\mathrm{y}}^{2} / \Omega_{0}^{2}$ against $\xi$ is shown in Fig. 3 . The temperature dependence of $\omega_{\mathrm{y}}^{2} / \Omega_{0}^{2}$ in this way arises from the fact that the shift of the atom adjoining a vacant site $\eta$, which is given by (30), increases with temperature through (34). 
In the next place, according to eq. (21), we discuss the influence of motion of atom 2 upon that of atom 1 adjoing a vacancy in the atomic chain shown in Fig. 4. In the chain, atoms 1, 2, $3, \cdots$ vibrate with the same basis frequency $\omega$, and it is the eigen frequency for atoms 2, 3, $4, \cdots$, but corresponds to that of forced oscillation for atom 1 which has the eigen frequency $\omega_{\mathrm{y}}$.

The motion of atom 2 is given by

$$
x=A \sin \omega t+B \sin 2 \omega t+C \sin 3 \omega t+\cdots \text {. }
$$

as described in $\S I I$, and the basis frequency $\omega$ depends upon temperature as shown in Fig. 3.

If the frequency $\omega$ of atom 2 differs from $\omega_{y}$ of atom $1, \omega_{y}$ is not affected by the motion of atom 2 , so that the temperature variation of $\omega_{y}$ can be given by (35). But we find $\omega=\omega_{y}$ at $\xi=0.0344$ from the intersection of the curves of $\omega^{2} / \Omega_{0}^{2}$ and $\omega_{y} / \Omega^{2}{ }_{0}$ shown in Fig. 3. There is a possibility that a sort of resonance happens between the vibrations of atom 1 and 2 at temperature $\xi=0.0344$.

The equation of motion (21) can be rewritten approximately from (29) neglecting small quantities of terms connecting with $y_{2}$ and $x^{2}$ as follows.

$$
\begin{aligned}
& \left(\ddot{y}_{0}+\omega_{\mathrm{y}}^{2} y_{0}+2 \theta_{0}^{2} x y_{0}\right)+\left(\ddot{y}_{1}+4 \omega_{\mathrm{y}}^{2} y_{1}+2 \theta_{0}^{2} x y_{1}\right) \\
& =\Omega_{0}^{2}\left(1-2 \frac{\theta_{0}^{2}}{\Omega_{0}^{2}} \eta\right) x,
\end{aligned}
$$

where $x$ is given by (36). But vibrational behaviors of $y_{0}$ and $y_{1}$ are independent of each other, and in the case of $\omega=\omega_{\mathrm{y}}$ vibrations $y_{0}$ and $y_{1}$ have the possibility of resonance with the terms $A \sin \omega t$ and $B \sin 2 \omega t$ in $x$, respectively, so the term including $y_{0}$ may be separated from that of $y_{1}$ as follows.

$$
\begin{aligned}
\ddot{y}_{0} & +\omega_{y}^{2}\left\{1+\frac{2 \theta_{0}^{2}}{\omega_{y}^{2}}(A \sin \omega t+B \sin 2 \omega t)\right\} y_{0} \\
= & \Omega_{0}^{2}\left(1-2 \frac{\theta_{0}^{2}}{\Omega_{0}^{2}} \eta\right) A \sin \omega t, \\
\ddot{y}_{1} & +4 \omega_{y}^{2}\left\{1+\frac{\theta_{0}^{2}}{2 \omega_{y}^{2}}(A \sin \omega t+B \sin 2 \omega t)\right\} y_{1} \\
= & \Omega_{0}^{2}\left(1-2 \frac{\theta_{0}^{2}}{\Omega_{0}^{2}} \eta\right) B \sin 2 \omega t .
\end{aligned}
$$

Each of eqs. (37) and (38) is a modified type of Mathieu's equation accompanied with the term of forced oscillation and a linear differential equation of $y$. Therefore, the solution may be given generally by a linear combination of terms connecting with the basis vibration of the eigen frequency $\omega_{\mathrm{y}}$ and the forced vibration of the frequency $\omega$. When the term of forced vibration is taken to be zero on the right hand side of eq. (37) or (38), each of these equations is reduced to Mathieu's equation, and the solutions $y_{0}$ and $y_{1}$ are non-periodic and unstable at

$$
\omega=2 \omega_{\mathrm{y}}, \omega_{\mathrm{y}}, \frac{2}{3} \omega_{\mathrm{y}}, \cdots,
$$

where a sort of resonance takes place. When the third term on the left hand side of eq. (37) or (38) is zero, these equations are turned into

$$
\begin{aligned}
& \ddot{y}_{0}+\omega_{\mathrm{y}}^{2} y_{0}=\Omega_{0}^{2}\left(1-2 \frac{\theta_{0}^{2}}{\Omega_{0}^{2}} \eta\right) A \sin \omega t, \\
& \ddot{y}_{1}+4 \omega_{\mathrm{y}}^{2} y_{1}=\Omega_{0}^{2}\left(1-2 \frac{\theta_{0}^{2}}{\Omega_{0}^{2}} \eta\right) B \sin 2 \omega t .
\end{aligned}
$$

The solutions $y_{0}$ and $y_{1}$ in the above equations come to be non-periodic at $\omega=\omega_{\mathrm{y}}$.

The frequencies $\omega_{\mathrm{y}}$ and $\omega$ depend upon temperature $\xi$ and in the temperature range below $\xi=0.0344$ at $\omega=\omega_{y}$, we can find

$$
1 \geq \frac{\omega^{2}}{\omega_{\mathrm{y}}^{2}}>\left(\frac{2}{3}\right)^{2}
$$

from Fig. 3. Therefore, the solution $y_{0}$ for eq. (37) and $y_{1}$ for (38) are supposed to turn out non-periodic and unstable at $\omega=\omega_{\mathrm{y}}$.

To discuss, at first, the behavior of $y_{0}$ in (37) in the case of $\omega=\omega_{y}$, we put

$$
y_{0}=a_{0}(t) \sin \omega_{\mathrm{y}} t+b_{0}(t) \cos \omega_{\mathrm{y}} t
$$

under the following restrictions (i) and (ii).

(i) $a_{0}(t)$ and $b_{0}(t)$ are functions which change very slowly over a longer time compared with the period of vibration $T=2 \pi / \omega_{y}$.

$$
\dot{a}_{0}(t) \sin \omega_{\mathrm{y}} t+\dot{b}_{0}(t) \cos \omega_{\mathrm{y}} t=0 .
$$

Substituting (39) for (37) under $\omega=\omega_{\mathrm{y}}$ and using (40), $\dot{a}_{0}(t)$ and $\dot{b}_{0}(t)$ are obtained in terms including $\sin \omega_{\mathrm{y}} t$ and $\cos \omega_{\mathrm{y}} t$. When the time average is taken over the period $T$ for these $\dot{a}_{0}(t)$ and $\dot{b}_{0}(t)$, it follows that 


$$
\dot{a}_{0}(t)=\frac{B a_{0}(t)}{2 \omega_{y}} \theta_{0}^{2},
$$

then, we have

$$
a_{0}(t)=\alpha \exp \left(-\frac{B \theta_{0}^{2}}{2 \omega_{y}} t\right)
$$

And also

$$
\dot{b}_{0}(t)=\frac{B \theta_{0}^{2}}{2 \omega_{y}} b_{0}(t)-\Omega_{0}^{2}\left(1-2 \frac{\theta_{0}^{2}}{\Omega_{0}^{2}} \eta\right) \frac{A}{2 \omega_{y}},
$$

then, it follows that

$$
b_{0}(t)=\left(1-2 \frac{\theta_{0}^{2}}{\Omega_{0}^{2}} \eta\right) \frac{A}{B} \frac{\Omega_{0}^{2}}{\theta_{0}^{2}}+\frac{2 \omega_{y}}{B \theta_{0}^{2}} \exp \left(\frac{B \theta_{0}^{2}}{2 \omega_{y}} t\right) \text {. }
$$

$B \theta_{0}^{2} t / 2 \omega_{y}$ in the above $a_{0}(t)$ and $b_{0}(t)$ is rewritten as

$$
B \frac{\theta_{0}^{2}}{2 \omega_{y}} t=B \frac{\theta_{0}^{2}}{\Omega_{0}^{2}} \frac{\Omega_{0}^{2}}{\omega_{y}^{2}} \frac{\omega_{y}}{2} t,
$$

where $B \theta_{0}^{2} / \Omega_{0}^{2}$ has been given in (12), and it takes a negative valve -0.0655 at $\xi=0.0344$. Consequently $a_{0}(t)$ increases with time, diverging to $\infty$, but the second term in $b_{0}(t)$ decreases rapidly with time, tending to zero. Thus $y_{0}$ in (39) turns out

$$
y_{0}=\alpha \exp \left(-\frac{1}{2} \frac{B \theta_{0}^{2}}{\omega_{\mathrm{y}}} t\right) \sin \omega_{\mathrm{y}} t,
$$

but the vibration becomes non-periodic and unstable. Next, the behavior of $y_{1}$ at $\omega=\omega_{y}$ is discussed from (38) by putting

$$
y_{1}=a_{1}(t) \sin 2 \omega_{\mathrm{y}} t+b_{1}(t) \cos 2 \omega_{\mathrm{y}} t
$$

and

$$
\dot{a}_{1}(t) \sin 2 \omega_{\mathrm{y}} t+\dot{b}_{1}(t) \cos 2 \omega_{\mathrm{y}} t=0 .
$$

$\dot{a}_{1}(t)$ and $\dot{b}_{1}(t)$ are evaluated in a similar way to the case of $y_{0}$ and it follows that

and

$$
2 \omega_{y} \dot{a}_{1}(t)=0
$$

$$
2 \omega_{y} \dot{b}_{1}(t)=-\frac{1}{2}\left(1-2 \frac{\theta_{0}^{2}}{\Omega_{0}^{2}} \eta\right) B \Omega_{0}^{2} .
$$

Then we have

$$
a_{1}(t)=C
$$

and

$$
b_{1}(t)=D-\left(1-2 \frac{\theta_{0}^{2}}{\Omega_{0}^{2}} \eta\right) \frac{B \Omega_{0}^{2}}{4 \omega_{y}} t,
$$

where $C$ and $D$ are constants independent of $t$. Since $B<0$ and $\left(1-2 \theta_{0}^{2} \eta / \Omega_{0}^{2}\right)=1-A^{2} \theta_{0}^{4} / \Omega_{0}^{4} \approx 1$, $b_{1}(t)$ increases with time $t$. One may take $C=D=0$ in this case, so that $y_{1}$ becomes

$$
y_{1}=-\left(1-2 \frac{\theta_{0}^{2}}{\Omega_{0}^{2}} \eta\right) \frac{B \Omega_{0}^{2}}{4 \omega_{y}} t \cos 2 \omega_{y} t,
$$

but $y_{1}$ tends toward unstable with increasing $t$. Thus the vibrational motion of atom 1 , which is given by $y=y_{0}+y_{1}$, is unstable due to the resonance at $\omega=\omega_{y}$, and leaving the equilibrium position it moves toward the vacant site.

Another atom 1' adjacent to the vacant site is in the same circustance as atom 1 in Fig. 4, so that it is displaced toward the vacant site until the distance between atoms 1 and $1^{\prime}$ comes nearly to $a$ which is the equilibrium distance between atoms. In succession atoms 2 and $2^{\prime}$ shift toward vacant sites which atoms 1 and $1^{\prime}$ leave behind, respectively, and atoms 3 and $3^{\prime}$ follow.

\section{Atomic Processes of Melting}

In this section we discuss the behaviours of atoms adjoining vacant sites in the three dimensional lattice with such as f.c.c. structure. There exist twelve atoms adjacent to a vacant site in the f.c.c. or h.c.p. lattice. Each of these atoms has tendency to move toward the vacant site due to the vibrational resonance at temperature of $\xi=0.0344$, however it is impossible for them to approach the vacant site simultaneously, owing to the repulsive force between one another. The phases of vibration in these atoms are considered to be different from one to another so that few atoms can be displaced leaving vacant sites behind from their normal sites into interstitial positions in f.c.c. lattice being squeezed in between normal atoms. Thus Frenkel defects consisting of vacant sites and interstitial atoms are formed in the lattice as a result of vibrational resonance. The successive formation of the secondary group of Frenkel defects follows the displacement of atoms adjoining vacant sites which are brought about by the formation of the first group of Frenkel defects. This process will be successively continued. Thus, a number of 
Frenkel pairs are accumulated in a crystalline domain around a vacancy through the chainreaction like spreading of vibrational resonance.

In closely packed structures there is little space available for interstitial atoms. The location of interstitial atoms sets up localized internal strains in the lattice, and also each of atoms around vacant sites produces a shift $\eta$ from the normal site. The shift $\eta$ becomes larger with increasing temperature as seen from eq. (30), but it is not necessarily equal for each of atoms around a vacant site, because of the repulsive force among one another. Accordingly the local disorderly arrangement of atoms around a vacant site and an interstitial atom is accompanied by the formation of a Frenkel defect at higher temperature. It has been shown from the pair correlation function evaluated by the computor simulation that when over about 0.4 Frenkel defects per atom are introduced into a domain with f.c.c. lattice at $0{ }^{\circ} \mathrm{K}$ its crystal array changes to the disordered arrangement ${ }^{(6)}$. At higher temperature of $\xi=0.0344 \eta$ increases so that a crystalline domain with less than 0.4 Frenkel defects per atom is also considered to change to the disordered state.

Now, we can define that the melting point $T_{\mathrm{m}}$ is given by the temperature at which the vibrational resonance takes place in a crystal and $T_{\mathrm{m}}$ is determined from the relation

$$
\frac{k T_{\mathrm{m}}}{m \Omega_{0}^{2}} \frac{\theta_{0}^{4}}{\Omega_{0}^{4}}=0.0344 .
$$

In the process of the solid-liquid transition at $T_{\mathrm{m}}$, a crystalline domain involving $l$ atoms around each of vacancies generated thermally changes to the disorderly arrangement of atoms through the accumulation of Frenkel defects generated by the chain-reaction like spreading of the vibrational resonance. These processes are expressed thermodynamically as follows.

$$
l\left(\mu_{\mathrm{R}}-\mu_{\mathrm{S}}\right)=\mu_{\mathrm{h}},
$$

where $\mu_{\mathrm{R}}, \mu_{\mathrm{S}}$ and $\mu_{\mathrm{h}}$ are the chemical potemtial of the disordered state, that of the crystalline state and the free energy of vacancy formation, respectively. From the above equation $l$ is estimated at the order of $10^{1} \sim 10^{2}$. The equilibrium number of vacancies in a crystal consisting of $N$ atoms is

$$
(\Delta \cdot N)_{\mathrm{m}}=N \exp \left(-\frac{\mu_{\mathrm{h}}}{k T_{\mathrm{m}}}\right) .
$$

However, the number of vacancies necessary for the change from the crystalline state to the disordered one is $(N / l)$, which is exceedingly larger than $(\Delta N)_{\mathrm{m}}$.

Simon's experimental equation (1) suggests that the volume change on melting originates from vacancies formed in the process of the solid-liquid transition at the melting point. The first term on the right hand side of eq. (1) corresponds to vacancies, wich account for the change from the crystalline to the random state, so that we can take

$$
\frac{N}{l}=30(\Delta N)_{\mathrm{m}},
$$

from which $l$ is evaluated.

\begin{tabular}{llllll} 
& $\mathrm{Al}$ & $\mathrm{Ag}$ & $\mathrm{Cu}$ & $\mathrm{Au}$ & $\mathrm{Pb}$ \\
\hline$l$ & 37 & 196 & 130 & 50 & 196
\end{tabular}

The greater part of vacancies necessary for the transition from the crystalline to the liquid state are supplied from vacancies generated in the crystal surface. It is more probable to generate vacancies in the surface of crystal. Surface atoms are supposed to be in similar circumstances to atoms adjacent to vacancies in the interior of crystal. At the melting temperature, some of these surface atoms jump from normal sites to new sites on the crystal surface or into the vapour phase owing to the vibrational resonance mainly. The vacancies which these surface atoms leave behind then migrate into the interior of crystal. Originating from the vibrational resonance of atoms adjoining some of these vacancies a large mount of Frenkel defects are produced in crystalline domains near the surface, and the crystalline array in the domains results in the random arrangement. Other vacancies travel through the disordered domain near the surface layer into crystalline domains remaining in the interior of crystal, and the random arrangement of atoms is produced in these domains. 
The free energy of such a disordered state is higher than that of the crystalline state owing to the increase in the internal energy mainly, so that it has a probability to turn to the ordered state. However, if $\alpha N$ vacancies are still more introduced from the surface into the disorderly arrangement of $N$ atoms, they will move about freely among the disorderly array of oscillating atoms through the successive jumping of neighbouring atoms to the vacant sites.

The phase in vibration is at random among atoms adjoining a vacancy so that any one of these atoms can jump easily to the vacant site. Such a jumping process of an atom is considered to be the transfer from its vibrational state to the translational motion. Thus the disorderly state accompanied by the free migration of $\alpha N$ vacancies corresponds to the state in which $N(1-\alpha)$ atoms are in the vibrational motion and $\alpha N$ atoms in the translational motion in the potential field of their neighbouring atoms arranged disorderly. Consequently the oscillatory motion of each atom is more or less coherent with that of its immediate neighbours, but the coherency of the oscillation for groups of atoms decreases rapidly with the increase in the distance from a central atom. The phase and the life time of such a coherent oscillation in each group of a central atom and its first neighbours are different from one to another.

The potential energy of individual atom due to the mutual interaction with its neighbours is not always equal among $N$ atoms on account of the random arrangement, so that the multiplicity of the energy level $W$ arises. The free energy of the disorderes state consisting of $N$ atoms and $\alpha N$ vacancies will decrease based on the increase in the entropy $k \log W$ originating from the multiplicity of the energy level. This disordered state is considered to be the liquid phase. Though the vacancy concentration $\alpha$ is determined from the equilibrium condition $F_{\mathrm{S}}=F_{\mathrm{L}}$ where $F_{\mathrm{S}}$ and $F_{\mathrm{L}}$ are the free energy of the crystalline and the liquid state, respectively, at the melting point, we may put $\alpha=0.033$ from the second term of eq. (1) which is the vacancy concentration necessary for the transition from the disordered to the liquid state.
For connecting the melting point $T_{\mathrm{m}}$ with the cohesive energy $L_{0}$ of the crystal we use Morse's potential

$$
\begin{aligned}
V= & -L_{0}\left[e^{-2 a\left(R-R_{0}\right)}-2 e^{-a\left(R-R_{0}\right)}\right] \\
= & -L_{0}+L_{0}\left[\left(a R_{0}\right)^{2}\left(\frac{R-R_{0}}{R_{0}}\right)^{2}\right. \\
& \left.-\left(a R_{0}\right)^{3}\left(\frac{R-R_{0}}{R_{0}}\right)^{3}+\cdots\right],
\end{aligned}
$$

where $L_{0}$ is the cohesive energy of crystal and is given by the latent heat of evaporation at $0{ }^{\circ} \mathrm{K}$ experimentally, and $a$ is the numerical constant characteristic of metals which is evaluated from the compressibility. From the comparison of eqs. (42), (20) and (22), we have

$$
\frac{k T_{m}}{m \Omega_{0}^{2}} \frac{\theta_{0}^{4}}{\Omega_{0}^{4}}=\frac{9}{8} \frac{k T_{m}}{L_{0}}=0.0344 .
$$

This relation is satisfied by many metals, as shown in the following Table 2 .

We can evaluate the latent heat of fusion and the entropy change on melting using the relation (43) and $\alpha=0.033$.

\section{v. Latent Heat of Fusion and Entropy Change of Melting}

\section{Distribution function}

There exist $Z_{0}$ nearest neighbouring atoms on the spherical surface of the radius $R_{0}$ around an atom in the crystalline state. $R_{0}$ is the distance from the atom to the minimum position of potential. But in the liquid state

Table 2 Cohesive energy $L_{0}$ and $(9 / 8)\left(N k T_{\mathrm{m}} / L_{0}\right)$.

\begin{tabular}{lccc}
\hline \hline & $L_{0}(\mathrm{kcal} / \mathrm{mol})$ & $T_{\mathrm{m}}(\mathrm{K})$ & $\frac{9}{8} \frac{N k T_{\mathrm{m}}}{L_{0}}$ \\
\hline $\mathrm{Al}$ & 67.6 & 933.3 & 0.0311 \\
$\mathrm{Cu}$ & 81.7 & 1356.5 & 0.0373 \\
$\mathrm{Ag}$ & 69.4 & 1233.95 & 0.0400 \\
$\mathrm{Au}$ & 90.7 & 1336.15 & 0.0331 \\
$\mathrm{~Pb}$ & 42.88 & 600.6 & 0.0315 \\
$\mathrm{Pt}$ & 127.00 & 2042.2 & 0.0362 \\
$\mathrm{Zr}$ & 139.03 & 2128.0 & 0.0344 \\
$\mathrm{Pd}$ & 110.0 & 1825.15 & 0.0373 \\
$\mathrm{Na}$ & 25.53 & 370.95 & 0.0326 \\
$\mathrm{~K}$ & 21.9 & 336.4 & 0.0345 \\
$\mathrm{Rb}$ & 17.09 & 321.7 & 0.0345 \\
\hline \hline
\end{tabular}


associated with the disorderly arrangement of atoms, the potential energy of each atom, $\varepsilon$, due to the mutual interaction with its neighbours is not always equal. Let $n_{1}, n_{2}, \cdots$, $n_{i}, \cdots$ be the numbers of atom having the potential energy $\varepsilon_{1}, \varepsilon_{2}, \cdots, \varepsilon_{i}, \cdots$, respectively. The potential energy $\varepsilon$ of a given atom depends upon the number of neighbouring atoms which are randomly distributed around it. So we can define $\bar{Z}(1-\alpha)$ neighbours around an atom which have practically the mutual interaction with it and are distributed in a spherical shell around a central atom. The potential energy of each atom depends on how these neighbours are distributed in the shell. $\alpha$ is the vacancy concentration and $\bar{Z}$ is the mean number of neighbours around an atom in the case of the disordered arrangement like an amorphous state produced by the introduction of vacancies $30(\Delta N / N)_{\mathrm{m}}$ into the crystalline state. However, the volume change $\Delta V / V$ due to the introduction of these vacancies is no more than about 0.1 so that $\bar{Z}$ is considered to be nearly equal to $Z_{0}$.

In the above energy distribution, the potential energy $\varepsilon_{i}$ is associated with the sum of pair potentials between a given atom and $\bar{Z}(1-\alpha)$ neighbours which have a given distribution in the spherical shell around it, and if the average of these pair potentials is denoted by $u^{\prime}\left(R_{i}\right), \varepsilon_{i}$ can be given by

$$
\varepsilon_{i}=\frac{\bar{Z}(1-\alpha)}{2} u^{\prime}\left(R_{i}\right) .
$$

This quation (44) is equivalent to the energy relation in the case when $\bar{Z}(1-\alpha)$ neighbouring atoms are found on the spherical surface of the radius $R_{i}$ around a given atom. However, as the probability that a given atom has the potential energy $\varepsilon_{i}$ is $n_{i} / N$, so in the system of $N$ atoms, the mean potential energy of each atom $\bar{\varepsilon}=\sum_{i} n_{i} / N \cdot \varepsilon_{i}$ depends upon the distribution of $\left(n_{i} / N\right) \bar{Z}(1-\alpha)$ neighbouring atoms around it. If $Z\left(R_{i}\right)$ is the number of neighbouring atoms found on a spherical surface of a radius $R_{i}$ around an atom, it follows that

$$
\sum_{R_{i}} Z\left(R_{i}\right)=\bar{Z}(1-\alpha),
$$

then we can take

$$
\frac{Z\left(R_{i}\right)}{\bar{Z}(1-\alpha)}=\frac{n_{i}}{N}
$$

and

$$
\sum_{R_{i}} \frac{Z\left(R_{i}\right)}{\bar{Z}(1-\alpha)}=\sum_{i} \frac{n_{i}}{N}=1 .
$$

When the pair potential of an atom is denoted by $u(R), \bar{\varepsilon}$ is given by

$$
\begin{aligned}
\bar{\varepsilon}=\sum_{i} \frac{n_{i}}{N} \varepsilon_{i} & =\frac{\bar{Z}(1-\alpha)}{2} \sum_{i} \frac{n_{i}}{N} u\left(R_{i}\right) \\
& =\frac{\bar{Z}(1-\alpha)}{2} \sum_{R_{i}} \frac{Z\left(R_{i}\right)}{\bar{Z}(1-\alpha)} u\left(R_{i}\right) \\
& =\frac{\bar{Z}(1-\alpha)}{2} \overline{u(R)},
\end{aligned}
$$

because the mean pair potential $\overline{u(R)}$ is given by

$$
\sum_{R_{i}} \frac{Z\left(R_{i}\right)}{\bar{Z}(1-\alpha)} u\left(R_{i}\right)=\sum_{i} \frac{n_{i}}{N} u\left(R_{i}\right)=\overline{u(R)} .
$$

Thus the energy distribution in the liquid state will be reduced to the distribution of neighbouring atoms around an atom.

The probability $Z(R) / \bar{Z}(1-\alpha)$ may be given by

$$
\frac{Z(R)}{\bar{Z}(1-\alpha)}=\frac{Z\left(R_{0}\right)}{\bar{Z}(1-\alpha)} \exp \left[-\frac{u(R)-u\left(R_{0}\right)}{k T_{m}}\right] .
$$

$Z\left(R_{0}\right) / \bar{Z}(1-\alpha)$ is the probability that an atom is found at the distance $R_{0}$ from a given atom, and is determined by solving the following equation

$$
\int_{\frac{R_{1}}{R_{0}}}^{\frac{R_{2}}{R_{0}}} 4 \pi\left(\frac{R}{R_{0}}\right)^{2} \frac{Z\left(R_{0}\right)}{\bar{Z}(1-\alpha)} \exp \left[-\frac{u(R)-u\left(R_{0}\right)}{k T_{m}}\right] d\left(\frac{R}{R_{0}}\right)=1,
$$

where integration limits $R_{1}$ and $R_{2}$ are the lower and the upper radius of the spherical shell, respectively. 
When Morse's potential is used for $u(R)$, we have the distribution function

$$
\frac{Z(R)}{\bar{Z}(1-\alpha)}=\frac{Z\left(R_{0}\right)}{\bar{Z}(1-\alpha)} \exp \left[-\frac{2 L_{0}}{Z_{0} k T_{m}}\left\{\left(a R_{0}\right)^{2}\left(\frac{R-R_{0}}{R_{0}}\right)^{2}-\left(a R_{0}\right)^{3}\left(\frac{R-R_{0}}{R_{0}}\right)^{3}\right\}\right] .
$$

In the above equation (50) we can put

and

$$
\bar{Z} \approx Z_{0}=12
$$

$$
\frac{L_{0}}{k T_{\mathrm{m}}}=32.7035
$$

from eq. (43).

The exponential factor in eq. (50) decreases rapidly with the increase in $\left(R-R_{0}\right) / R_{0}$ and is practically zero in the neighbourhood of $\left(R-R_{0}\right) / R_{0} \approx-0.14$ and +0.3 , so that the limit of integration in eq. (49) may be taken as $R_{1} / R_{0}=-\infty$ and $R_{2} / R_{0}=+\infty$, and it folows that

$$
\int_{-\infty}^{+\infty} 4 \pi \frac{Z\left(R_{0}\right)}{\bar{Z}(1-\alpha)}\left(\frac{R}{R_{0}}\right)^{2} \exp \left[-\frac{2 L_{0}}{Z_{0} k T_{m}}\left\{\left(a R_{0}\right)^{2}\left(\frac{R-R_{0}}{R_{0}}\right)^{2}-\left(a R_{0}\right)^{3}\left(\frac{R-R_{0}}{R_{0}}\right)^{3}\right\}\right] d\left(\frac{R}{R_{0}}\right)=1 .
$$

When we put for simplicity

$$
\begin{aligned}
& \frac{R-R_{0}}{R_{0}}=x, \quad \beta=\frac{2 L_{0}}{Z_{0} k T_{m}}\left(a R_{0}\right)^{2}, \\
& \delta=\frac{2 L_{0}}{Z_{0} k T_{m}}\left(a R_{0}\right)^{3},
\end{aligned}
$$

the above equation is written as

$$
4 \pi \frac{Z\left(R_{0}\right)}{\bar{Z}(1-\alpha)} \int_{0}^{\infty} 2\left(1+x^{2}+2 \delta x^{4}\right) e^{-\beta x^{2}} d x=1
$$

$Z\left(R_{0}\right) / \bar{Z}(1-\alpha)$ is evaluatated by using the quantities $a, R_{0}$ and $a R_{0}$ given in Table 3 . $\left(Z\left(R_{0}\right) / \bar{Z}(1-\alpha)\right)$ is shown in Table 4 for several metals.

\section{Latent heat of fusion $\Delta E$}

In the crystalline state consisting of $N$ atoms, every atom is in vibration at the lattice site which corresponds to the minimum position of potential. Then the total energy of $N$ atoms at the melting temperature is given by

$$
E_{\mathrm{s}}=-N L_{0}+3 N k T_{\mathrm{m}} .
$$

It is considered that in the liquid state each of $N(1-\alpha)$ atoms is in the vibrational motion under the mutual interaction with $\bar{Z}(1-\alpha)$
Table 3 Constants concerned in energy relation.

\begin{tabular}{lccc}
\hline \hline & $a\left(\AA^{-1}\right)$ & $R_{0}(\AA)$ & $a R_{0}$ \\
\hline $\mathrm{Al}$ & 1.2043 & 2.8635 & 3.4485 \\
$\mathrm{Cu}$ & 1.4067 & 2.5560 & 3.5955 \\
$\mathrm{Ag}$ & 1.3855 & 2.8894 & 4.0032 \\
$\mathrm{Au}$ & 1.5724 & 2.8839 & 4.5346 \\
\hline \hline
\end{tabular}

Table $4 \quad Z\left(R_{0}\right) / \bar{Z}(1-\alpha)$ at $T_{\mathrm{m}}$.

\begin{tabular}{ccccc}
\hline \hline & $\mathrm{Al}$ & $\mathrm{Cu}$ & $\mathrm{Ag}$ & $\mathrm{Au}$ \\
\hline$\frac{Z\left(R_{0}\right)}{\bar{Z}(1-\alpha)}$ & 0.3324 & 0.3478 & 0.3905 & 0.4462 \\
\hline \hline
\end{tabular}

neighbouring atoms in the disorderly arrangement and $\alpha N$ atoms are in the translational motion, where $\alpha$ is the vacancy concentration.

The kinetic energy of these $N(1-\alpha)$ atoms is given by

$$
\frac{3}{2} N(1-\alpha) k T_{\mathrm{m}},
$$

but the potential energy of each atom due to the mutual interaction with the neighbours is not always equal, so if the averaged pair potential is $\overline{u(R)}$, the potential energy of $N(1-\alpha)$ atoms is written as

$$
\frac{1}{2} \bar{Z}(1-\alpha) N(1-\alpha) \overline{u(R)} \text {, }
$$

where

$$
\overline{u(R)}=-\frac{2}{Z_{0}} L_{0}+\frac{2}{Z_{0}} L_{0}\left\{\left(a R_{0}\right)^{2} \overline{\left(\frac{R-R_{0}}{R_{0}}\right)^{2}}-\left(a R_{0}\right)^{3} \overline{\left(\frac{R-R_{0}}{R_{0}}\right)^{3}}\right\}
$$


As the motion of each of $\alpha N$ atoms is one dimensional translation toward a neighbouring vacancy in the potential field of its neighbours, so the kinetic energy of $\alpha N$ atoms is given by

$$
\frac{1}{2} \alpha N k T_{\mathrm{m}}
$$

and the potential energy may be approximated as

$$
\frac{\bar{Z}(1-\alpha)}{2} \alpha N \overline{u^{\prime}}
$$

where $\overline{u^{\prime}}$ is considered to be nearly equal to $\bar{u}$. Thus the total potential energy of liquid state is written as

$$
\frac{\bar{Z}(1-\alpha)}{2} N(1-\alpha) \bar{u}+\frac{\bar{Z}(1-\alpha)}{2} \alpha N \overline{u^{\prime}} \fallingdotseq \frac{\bar{Z}(1-\alpha)}{2} N \bar{u},
$$

and the total kinetic energy is

$$
\frac{3}{2} N(1-\alpha) k T_{\mathrm{m}}+\frac{1}{2} \alpha N k T_{\mathrm{m}}=\left(\frac{3}{2}-\alpha\right) N k T_{\mathrm{m}} .
$$

Then, the total energy of liquid state $E_{\mathrm{L}}$ is given by

$$
E_{\mathrm{L}}=N(1-\alpha) \frac{\bar{Z}}{2} \bar{u}+\left(\frac{3}{2}-\alpha\right) N k T_{\mathrm{m}} .
$$

The latent heat of fusion $\Delta E$ is then

$$
\begin{aligned}
\Delta E & =E_{L}-E_{S} \\
& =\alpha N L_{0}+(1-\alpha) N L_{0}\left\{\left(a R_{0}\right)^{2} \overline{\left(\frac{R-R_{0}}{R_{0}}\right)^{2}}-\left(a R_{0}\right)^{3} \overline{\left(\frac{R-R_{0}}{R_{0}}\right)^{3}}\right\}-\left(\frac{3}{2}+\alpha\right) N k T_{m} .
\end{aligned}
$$

When $L_{0}=32.7035 k T_{\mathrm{m}}$ and $\alpha=0.033$ are substituted for eq. (54), $\Delta E$ is written in the term of $k T_{\mathrm{m}}$. The mean values $\overline{\left(\left(R-R_{0}\right) / R_{0}\right)^{2}}$ and $\overline{\left(\left(R-R_{0}\right) / R_{0}\right)^{3}}$ are evaluated using the distribution function (50) and are obtained from the following equation

$$
\overline{\left(\frac{R-R_{0}}{R_{0}}\right)^{n}}=4 \pi \frac{Z\left(R_{0}\right)}{\bar{Z}(1-\alpha)} \int_{0}^{\infty} 2 x^{n}\left(1+x^{2}+2 \delta x^{4}\right) e^{-\beta x^{2}} d x .
$$

$\Delta E$ (cal) evaluated from eq. (54) and $\Delta E$ (obs) are shown in the Table 5 .

When we take the average of these values estimated above, $\Delta E$ is written as

$$
\Delta E=1.173 N k T_{\mathrm{m}}=2.346 T_{\mathrm{m}} .
$$

We can see from Fig. 5 that the linear relation

Table 5 Latent heat of fusion $\Delta E(\mathrm{kcal} / \mathrm{mol})$.

\begin{tabular}{lrll}
\hline \hline & $T_{\mathrm{m}}(\mathrm{K})$ & $\Delta E(\mathrm{cal})$ & $\Delta E(\mathrm{obs})$ \\
\hline $\mathrm{Al}$ & 933 & $\left(1.1882 N k T_{\mathrm{m}}\right)$ & \\
& & 2.2177 & $2.046 \sim 2.55$ \\
$\mathrm{Cu}$ & 1365 & $\left(1.1815 N k T_{\mathrm{m}}\right)$ & \\
& & 3.2053 & 3.1309 \\
$\mathrm{Ag}$ & 1234 & $\left(1.1685 N k T_{\mathrm{m}}\right)$ & \\
& & 2.8838 \\
$\mathrm{Au}$ & 1336 & $\left(1.1540 N k T_{\mathrm{m}}\right)$ & $2.50 \sim 2.748$ \\
& & 3.0839 & 2.956 \\
\hline \hline
\end{tabular}

between $\Delta E$ and $T_{\mathrm{m}}$ in eq. (55) is satisfied for many metals with the f.c.c. and h.c.p. structure.

\section{Entropy change}

The entropy change on the transition from the crystalline to the liquid state is considered to be contributed from the change in the thermal entropy and in the entopy originating from the multiplicity of potential energy level.

The thermal entropy in the crystalline state composed of $N$ atoms is associated with the vibrational motion of atom in the potential field of its neighbours and is given by $3 \mathrm{Nk}$ from the equipartition law. In the liquid state $N(1-\alpha)$ atoms are in the vibrational motion and $\alpha N$ atoms are in the translational motion which is one dimensional, and then the ther- 


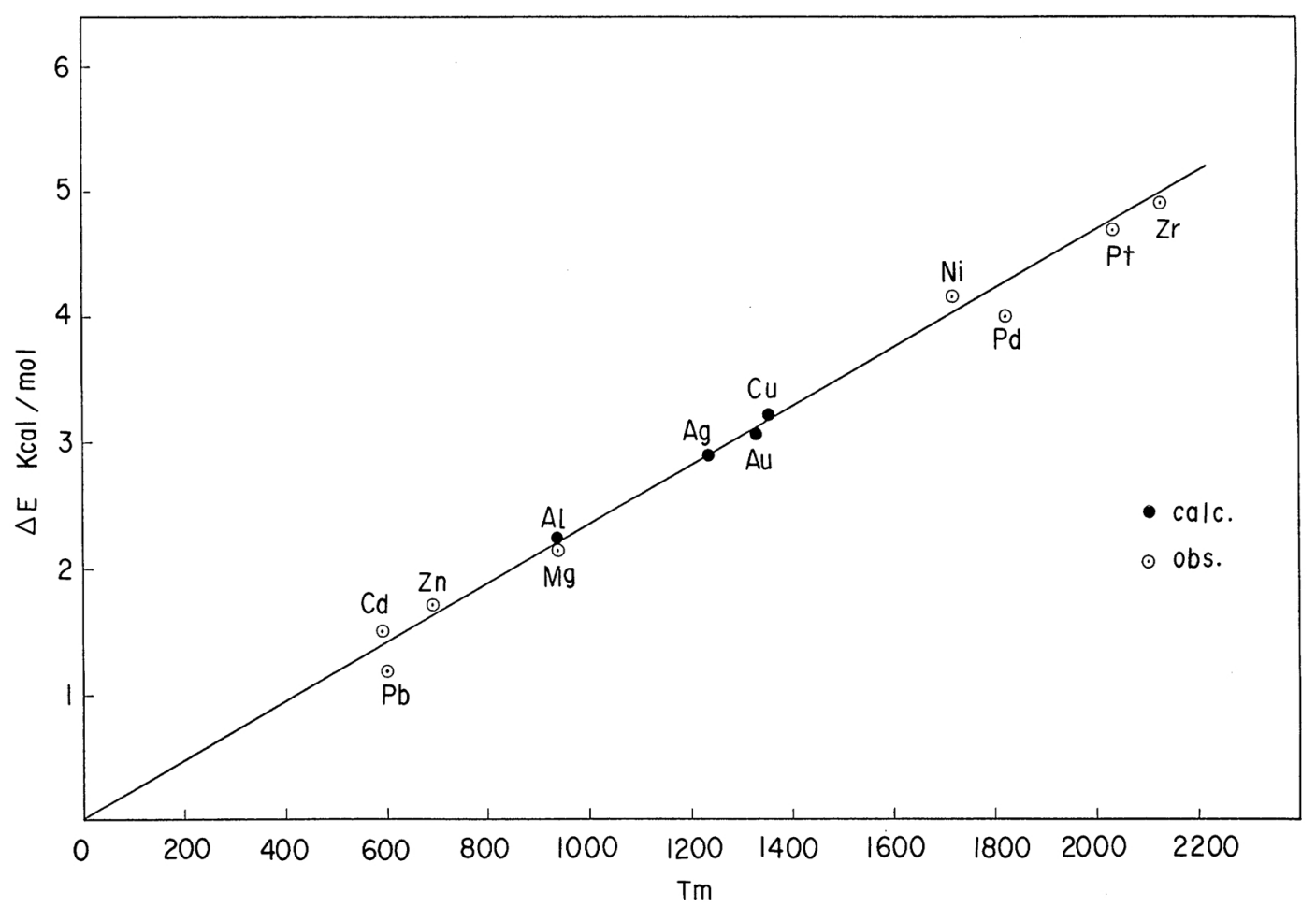

Fig. 5 Latent heat of fusion for metals with f.c.c. and h.c.p. structure. $\Delta E=2.346 T_{\mathrm{m}}(\mathrm{kcal} / \mathrm{mol})$.

mal entropy is given by

$$
3 N(1-\alpha) k+\alpha N k=(3-2 \alpha) N k,
$$

so that the change in the thermal entropy on melting is

$$
\Delta S_{1}=(3-2 \alpha) N k-3 N k=-2 \alpha N k .
$$

The potential energy of atom is not always the same due to the disorderly arrangement of atoms in the liquid. There exist $n_{1}, n_{2}, \cdots$, $n_{i}, \cdots$ atoms having the potential energy $\varepsilon_{1}$, $\varepsilon_{2}, \cdots, \varepsilon_{i}, \cdots$, respectively, and then the total potential energy of liquid state $N \bar{\varepsilon}$ is written as

$$
N \bar{\varepsilon}=\sum_{i} n_{i} \varepsilon_{i}
$$

Consequently the multiplicity of the potential energy level $N \bar{\varepsilon}$ is given by

$$
W_{L}=\frac{N !}{\prod_{i} n_{i} !} .
$$

On the other hand, as in the crystalline state every atom can be considered to have the same energy, so the multiplicity $W_{\mathrm{s}}$ is equal to 1 . Thus the entorpy change originating from the multiplicity of the energy level $\Delta S_{2}$ is given by

$$
\begin{aligned}
\Delta S_{2} & =k \log _{e} W_{L} \\
& =-N k \sum_{i} \frac{n_{i}}{N} \log _{e} \frac{n_{i}}{N} .
\end{aligned}
$$

From eq. (45) $\Delta S_{2}$ is rewritten as

$$
\Delta S_{2}=-N k \sum_{R_{i}} \frac{Z\left(R_{i}\right)}{\bar{Z}(1-\alpha)} \log _{e} \frac{Z\left(R_{i}\right)}{\bar{Z}(1-\alpha)} .
$$

If the above equation is expressed in the integral form, it follows from eq. (48) that

$$
\begin{aligned}
\Delta S_{2} & =-N k \int_{-\infty}^{\infty} 4 \pi\left(\frac{R}{R_{0}}\right)^{2} \frac{Z(R)}{\bar{Z}(1-\alpha)} \log _{e} \frac{Z(R)}{\bar{Z}(1-\alpha)} d\left(\frac{R}{R_{0}}\right) \\
& =-N k \log _{e} \frac{Z\left(R_{0}\right)}{\bar{Z}(1-\alpha)} \int_{-\infty}^{\infty} 4 \pi\left(\frac{R}{R_{0}}\right)^{2} \frac{Z(R)}{\bar{Z}(1-\alpha)} d\left(\frac{R}{R_{0}}\right)
\end{aligned}
$$




$$
\begin{aligned}
& +N k \int_{-\infty}^{\infty} 4 \pi\left(\frac{R}{R_{0}}\right)^{2} \frac{u(R)-u\left(R_{0}\right)}{k T_{m}} \frac{Z\left(R_{0}\right)}{\bar{Z}(1-\alpha)} \exp \left\{-\frac{u(R)-u\left(R_{0}\right)}{k T_{m}}\right\} d\left(\frac{R}{R_{0}}\right) \\
= & -N k \log _{e} \frac{Z\left(R_{0}\right)}{\bar{Z}(1-\alpha)}+N k\left\{\frac{u(R)-u\left(R_{0}\right)}{k T_{m}}\right\},
\end{aligned}
$$

where from eq. (53)

$$
\overline{\left\{\frac{u(R)-u\left(R_{0}\right)}{k T_{m}}\right\}}=\frac{2 L_{0}}{Z_{0} k T_{m}}\left\{\left(a R_{0}\right)^{2} \overline{\left(\frac{R-R_{0}}{R_{0}}\right)^{2}}-\left(a R_{0}\right)^{3} \overline{\left(\frac{R-R_{0}}{R_{0}}\right)^{3}}\right\} .
$$

Thus the entropy change on melting $\Delta S$ is given by

$$
\begin{aligned}
\Delta S & =\Delta S_{1}+\Delta S_{2} \\
& =\left[-\log _{e} \frac{Z\left(R_{0}\right)}{\bar{Z}(1-\alpha)}+\frac{2 L_{0}}{Z_{0} k T_{m}}\left\{\left(a R_{0}\right)^{2} \overline{\left(\frac{R-R_{0}}{R_{0}}\right)^{2}}-\left(a R_{0}\right)^{3} \overline{\left(\frac{R-R_{0}}{R_{0}}\right)^{3}}\right\}-2 \alpha\right] N k,
\end{aligned}
$$

where $Z\left(R_{0}\right) / \bar{Z}(1-\alpha)$ and $a R_{0}$ are given in Table 4 and 3 , respectively. When $L_{0} / k T_{\mathrm{m}}$ $=32.7035$ and $\alpha=0.033$ are substituted for eq. (59), $\Delta S$ (cal) and $\Delta S$ (obs) are shown in Table 6.

The average of $\Delta S$ (cal) is

$$
\Delta S=1.190 N k
$$

which is compared with

$$
\frac{\Delta E(\mathrm{cal})}{T_{\mathrm{m}}}=1.173 N k
$$

in eq. (55).

Table 6 Entropy change $\Delta S / N k$.

\begin{tabular}{lllll}
\hline \hline & $\mathrm{Al}$ & $\mathrm{Cu}$ & \multicolumn{1}{c}{$\mathrm{Ag}$} & \multicolumn{1}{c}{$\mathrm{Au}$} \\
\hline$\Delta S$ (cal) & 1.3185 & 1.272 & 1.1538 & 1.0180 \\
$\Delta S$ (obs) & 1.39 & 1.16 & 1.13 & 1.11 \\
\hline \hline
\end{tabular}

\section{Conclusion}

(1) The solid-liquid phase transition proceeds in two stages of the change from the crystalline to the disordered state and that from the disordered to the liquid state through vacancy mechanisms.

(2) These vacancy mechanisms are understood from the behaviours of atoms adjoining a vacancy and those apart from it, which are in anharmonic vibration at lattice sites.

(3) There exists a definite temperature $T^{*}$ at which the resonance arises between the vibrations of atoms described above.
(4) A number of Frenkel defects are generated in avalanche likesiwe in a crystalline domain around a vacancy due to the vibrational resonance at $T^{*}$, and the domain results in the random arrangement of atoms.

(5) Vacancy generation becomes more probable in the surface layer of crystal at $T^{*}$, then domains near the surface layer change into the disordered state by some of surface vacancies, and others diffuse into the interior of lattice.

(6) Vacancies can move about easily in the disordered arrangement of atoms, and this corresponds to that atoms of the identical concentration with vacancies change from the vibrational to the translational motion.

(7) Liquid is the state in which the vibrational motion coexists with the translational one. The temperature $T^{*}$ at which the resonance arises in the atomic vibration is the melting point $T_{\mathrm{m}}$.

(8) The latent heat of fusion and the entropy change on melting were evaluated according to the above atomic processes.

\section{REFERENCES}

(1) O. G. Peterson, D. Batchelder and R. O. Simons: Phil. Mag., 12 (1965), 1193.

(2) H. Kleinert: Phys Lett., 91A (1982), 295; Lett. Nuovo Cimento, 37 (1983), 295.

(3) S. Ami. T. Hofsass and R. Horoley: Phys. Lett., 101 (1984), 145.

(4) V. G. Chudinov and V. I. Protasov: Phys. Stat. Sol., (a) 85 (1984), 105.

(5) R. O. Simons: J. Phys. Soc. Japan 18. Suppl. II, (1963), 172.

(6) K. Maeda and S. Takeuchi: Phil. Mag., B52 (1985), 955. 